\title{
Minimal Order Observers for Estimating Linear Functions of a State Vector
}

\author{
JOHN B. MOORE, MEMBER, IEEE, AND GERARD F. LEDWICH, STUDENT MEMBER, IEEE
}

\begin{abstract}
This paper studies the necessary and sufficient conditions for a pth-order observer to observe linear functions of the states of a linear dynamical system. The conditions are a set of multivariable polynomial equations which must be satisfied for some variable set in order for a $p$ th-order observer to exist. It is possible to test for the existence of such a variable set in a finite number of steps via decision methods and thereby to construct an observer with the aid of polynomial factorization. To minimize the computational effort, the necessary and sufficient conditions are expressed in terms of the minimum number of variables.
\end{abstract}

\section{INTRODUCTION}

SINCE the problem addressed in this paper is the $\boldsymbol{S}_{\text {application of decision methods to minimal order }}$ observer design, the relevant perspectives of each of these topics is now reviewed.

1) Observers: For a dynamical system with input $u(\cdot)$, states $x(\cdot)$, and outputs $y(\cdot)$, recall that an observer is simply any asymptotically stable dynamical system driven from $u(\cdot)$ and $y(\cdot)$ with outputs $w(\cdot)$ satisfying

$$
\lim _{t \rightarrow \infty}\left[w(t)-K^{\prime} x(t)\right]=0
$$

for some specified $K^{\prime}$ (sometimes the identity matrix). Since the introduction of the Kalman observer [1] for estimating the states of an $n$ th-order linear system using an $n$ th-order observer of arbitrary asymptotically stable dynamics, efforts have been directed to build observers of lower order. Luenberger observers have arbitrary asymptotically stable dynamics and are of order $(n-m)$ where $m$ is the number of independent system outputs [1], [2]. These also estimate the state vector of an $n$ th-order linear system. A further reduction in the order of the observer is sometimes possible when estimating a linear functional of the state vector, as in the realization of the control law $K^{\prime} x$. It has been shown by Luenberger that it is always possible to estimate a scalar $K^{\prime} x$ using an observer with arbitrary asymptotically stable dynamics of order $\nu_{0}-1$ where $\nu_{0}$ is the observability index of the signal model. An obvious extension of this result is that an $r$-vector $K^{\prime} x$ can be estimated with $r$ such observers. However it is well known that if the observer dynamics are not fully specified, $r\left(\nu_{0}-1\right)$ may not represent the minimal order of an observer for estimating $K^{\prime} x$. On the

Manuscript received January 9, 1975; revised May 6, 1975. Paper recommended by J. B. Pearson, Chairman of the IEEE S-CS Linear Systems Committee. This research was supported by the Australian Research Grants Committee and the Radio Research Board.

J. B. Moore is with the Department of Electrical Engineering, University of Newcastle, Newcastle, New South Wales, Australia.

G. F. Ledwich is with the Department of Electrical Engineering, University of Newcastle, Newcastle, New South Wales, Australia. other hand, there is clearly a lower limit for the order of the observer of $(r-m)$.

Necessary and sufficient conditions for a $p$ th-order observer to estimate $K^{\prime} x$ where the observer dynamics are not specified are well known [2], [3], but to test these conditions using decidability theory and to construct the observer with the aid of polynomial factorization, extensive computing is required [4]. Fortmann and Williamson [3] showed that these conditions for the single-output case, are equivalent to a much simpler set of conditions. However, for the multiple-output case their results yielded nonminimal observers. Moore extended the results of [3] to the case when no direct feedthrough is permitted in the observer. This case is useful when it is desired that the measurements themselves have some filtering [4].

2) Decision Methods: Consider the equalities $p_{1}(v)=0$ and inequalities $p_{2}(v)<0$ where $p_{1}$ and $p_{2}$ are each a set of real multivariable polynomials in $v$ (polynomials in each of the components of $v$ ). The decision methods of [5]-[7] determine in a finite number of steps with each step involving only rational operations whether or not a vector $v$ exists such that $p_{1}(v)=0$ and $p_{2}(v)<0$. Unfortunately, the number of steps increases exponentially with the number of unknowns (elements of $v$ ) and also the number of inequalities. Reference [5] also provides methods for determining solutions $v$ which satisfy the equalities and inequalities - given of course that such $v$ are known to exist. These later methods involve polynomial factorization and thus in theory require an infinite number of steps.

Anderson et al. [5] show how decision techniques can be applied to, among other problems, minimal-order observer design for estimating $K^{\prime} x$. However, in their formulation there are a large number of unknowns and inequalities - at least for nontrivial problems.

\section{Description of Paper}

This paper explores necessary and sufficient conditions for the existence of a $p$ th-order observer for estimating linear functions of the state of a linear dynamical system involving as few as possible unknowns and inequalities. The conditions are manipulated to be suitable to the application of decision methods for a minimal-order observer design. Section II presents preliminary material including some results from [3]. Section III presents some immediate simplifications to the observer decision problem by suitable selection of observer and model coordinate basis. Section IV presents a series of properties of observers which are used in Section $\mathrm{V}$ to develop the key theorems 
which are observer existence conditions involving the minimum of unknowns. Section VI gives brief conclusions from the work.

\section{Preliminary Material}

Consider a linear time-invariant system described by the state equations

$$
\begin{aligned}
& \dot{x}(t)=F x(t)+G u(t) \\
& y(t)=H^{\prime} x(t)
\end{aligned}
$$

with $m$-vector output $\gamma, n$-vector states $x$, and input $u$ of arbitrary dimension. Assume without loss of generality that the above system is completely controllable, completely observable, and that $H$ and $G$ are of full rankthus $\left(H^{\prime} H\right)^{-1}$ exists.

A key result concerning observers of $K^{\prime} x$ (for $K^{\prime}$ an $r \times n$ matrix) discussed in [3] is now stated as a theorem.

Theorem 2.1: For the signal model (2.1) and (2.2):

1) Necessary and sufficient conditions for the existence of a linear, asymptotically stable, completely observable, $p$ th order observer estimates $K^{\prime} x$ from $u(\cdot)$ and $y(\cdot)$ are that there exist matrices $[A, B, C, E, T]$ of appropriate dimensions such that $[A, C]$ is completely observable,

$$
\begin{aligned}
& K^{\prime}=C^{\prime} T^{\prime} \text { for } E \text { constrained to be zero } \\
& K^{\prime}=C^{\prime} T^{\prime}+E H^{\prime} \text { for } E \text { unconstrained }
\end{aligned}
$$$$
T^{\prime} F-A T^{\prime}=B H^{\prime}
$$

and the eigenvalues of $A$, denoted $\lambda_{i}[A]$, are strictly negative (or equivalently for this latter condition, that Condition $\mathrm{Cl}$ below is satisfied).

2) The conditions $\mathrm{Cl},(2.3)$, and (2.4) are of themselves sufficient conditions for the existence of a $p$ th-order observer (not necessarily observable).

3) With $A, B, T, C$, and $E$ chosen to satisfy Cl, (2.3), and (2.4), a $p$ th-order observer is

$$
\begin{aligned}
& \dot{z}(t)=A z(t)+B y(t)+T^{\prime} G u(t) \\
& w(t)=C^{\prime} z(t)+E y(t) .
\end{aligned}
$$

Condition Cl: For the polynomial

$$
|s I-A|=\beta(s)=\beta_{0}+\beta_{1} s+\cdots+\beta_{p-1} s^{p-1}+\beta_{p} s^{p}=0
$$

with $\beta_{p}=1$, the $p$ Hurwitz determinants $\Delta_{i}^{\beta}$ satisfy the inequalities $\Delta_{i}^{\beta}>0$ for $i=1,2, \cdots p$ where $\Delta_{1}^{\beta}=\left|\beta_{p-1}\right|$,

$$
\Delta_{2}^{\beta}=\left[\begin{array}{ll}
\beta_{p-1} & \beta_{p-3} \\
1 & \beta_{p-2}
\end{array}\right], \quad \Delta_{3}^{\beta}=\left[\begin{array}{lll}
\beta_{p-1} & \beta_{p-3} & \beta_{p-5} \\
1 & \beta_{p-2} & \beta_{p-4} \\
0 & \beta_{p-1} & \beta_{p-3}
\end{array}\right] \text { etc. }
$$

or equivalently that $\beta_{i}>0$ for $i=0,1,2, \cdots p$ and $\Delta_{2 i+j}>0$ for $i=1,2, \cdots$ and $j=0$ or 1 .

Proof: The above theorem has been proven in [3]. Here we briefly outline an alternative proof of necessity of the conditions (2.3) and (2.4) which is possibly more direct than that of [3]. First, note that the observer (2.5) and
(2.6) given in terms of $B, T, C^{\prime}$, and $E$ is the most general $p$ th-order linear system that can be employed as an observer. The necessary and sufficient conditions for this to be an asymptotically stable observer of $K^{\prime} x$ is that $\mathrm{C} 1$ holds and $\lim _{t \rightarrow \infty}\left(w-K^{\prime} x\right)=0$. Since we are working with time-invariant systems the latter equality is equivalent to the following transfer function equality.

$$
\begin{aligned}
K^{\prime}(s I-F)^{-1} G & =\left[C^{\prime}(s I-A)^{-1} T(s I-F)\right. \\
& \left.+\left[E H^{\prime}+C^{\prime}(s I-A)^{-1} B H^{\prime}\right]\right](s I-F)^{-1} G
\end{aligned}
$$

where of course the right-hand side expression is the transfer function of a composite system (including observer) with input $u(t)$ and output $w(t)$.

Performing a partial fraction expansion using the identity $(s I-X)^{-1}=\left(s^{-1}+X s^{-2}+X^{2} s^{3}+\cdots\right)$ and equating the coefficient of $s^{-n}$ to zero yields

$$
\left[\begin{array}{l}
T\left[C A^{\prime} C\left(A^{2}\right)^{\prime} C \cdots\left(A^{n-1}\right)^{\prime} C\right] \\
+\left(H B^{\prime}-F^{\prime} T\right)\left[0 C(A)^{\prime} C \cdots\left(A^{n-2}\right)^{\prime} C\right] \\
+[(H E-K) 00 \cdots 0]
\end{array}\right]\left[\begin{array}{c}
F^{n-1} G \\
\cdot \\
\cdot \\
F G \\
G
\end{array}\right]=0
$$

Now defining controllability and observibility matrices

$$
\begin{aligned}
\bar{Q} & =\left[G^{\prime}\left(F^{n-1}\right)^{\prime} \cdots G^{\prime} F G^{\prime}\right] \\
Q^{\prime} & =\left[C A^{\prime} C \cdots\left(A^{p-1}\right)^{\prime} C\right] .
\end{aligned}
$$

This condition is equivalent to the conditions $\left(H E^{\prime}-K+\right.$ $T C) \bar{Q}^{\prime}=0$ and $\left(T A^{\prime}+H B^{\prime}-F^{\prime} T\right)\left[Q^{\prime}: X\right] \bar{Q}^{\prime}=0$ for some matrix $X$. Now clearly with $[F, G]$ completely controllable and $[A, C]$ completely observable, $\bar{Q}$ and $\left[Q^{\prime}: X\right]$ are of full rank and these equations are equivalent to (2.3) and (2.4). Necessity of (2.3) and (2.4) is thereby established. Note that if $[A, C]$ is not completely observable and or $[F, G]$ is not complete controllability, the conditions (2.3) and (2.4) are sufficient conditions for the existence of a $p$ th-order observer of $K^{\prime} x$ and our proof is complete.

\section{Comments}

1) Of course with the condition (2.4) satisfied

$$
\frac{d}{d t}\left(T^{\prime} x-z\right)=A\left(T^{\prime} x-z\right)
$$

and, given $\mathrm{Cl}$,

$$
\lim _{t \rightarrow \infty}\left(T^{\prime} x-z\right)=0
$$

2) The direct feedthrough term $E$ may be constrained to zero in order to prevent additive measurement noise from passing unfiltered into the estimate of $K^{\prime} x$. This case will be referred to loosely as the $E=0$ case. The usual case when $E$ is unconstrained will be loosely referred to as the case $E \neq 0$.

3) Luenberger [2] introduces the assumption that the observer does not share any eigenvalues in common with 
the signal model. This allows a unique solution of $(2.4)$ for $T$. Here, we avoid such restrictions.

4) The observability condition on the pair $A, C$ can be expressed as an inequality $\left|Q^{\prime} Q\right| \neq 0$ where $Q$ is the observability matrix. Actually if the conditions (2.1), (2.3), and (2.4) are tested for observers of orders, $0,1,2, \cdots$ in sequence, the observability condition need not be tested. To see this, assume that the necessary and sufficient conditions are not satisfied for observers of orders 0,1 , $2, \cdots, p-1$. Also assume that the sufficient conditions $\mathrm{Cl}$, (2.3), and (2.4) are satisfied for observers of order $p$, but $\left|Q^{\prime} Q\right|=0$. Then, an observer of order $p$ exists which is not completely observable and as a consequence, a reduced-order observer would be obtained by removing the unobservable states. But this contradicts the first assumption and the desired result is established.

5) Reference [5] points out that the conditions $\mathrm{Cl}$, (2.3), and (2.4) are in fact $(p+r) n$ multivariable polynomial equations, and $p$ inequalities involving $p^{2}+p(n+$ $m+r)+m r$ unknown elements of $A, B, C, E, T$. Moreover, the question of whether or not a $p$ th-order observer exists, can be determined in a finite number of rational steps by the application of the methods of [6], [7]. With knowledge that such an observer exists, the observer can be found by algorithms involving polynomial factorization. The algorithms unfortunately suffer from a "curse of dimensionality." That is, the number of unknown elements and the number of inequalities have an exponential or factorial effect on calculation time.

6) A result which is readily verified (but apparently not observed by the earlier authors in the field) is that the solution of condition (2.4) for $T^{\prime}$ and $B$ is

$T^{\prime}=-\left[\tilde{B}^{\prime} A \tilde{B}^{\prime} A^{2} \tilde{B}^{\prime} \cdots A^{n-1} \tilde{B}^{\prime}\right]\left[\begin{array}{cccc}\alpha_{1} I_{m} \cdots \alpha_{n-m} I_{m} & \alpha_{n} I_{m} \\ \alpha_{2} I_{m} \cdots & \alpha_{n} I_{m} & 0 \\ \cdot & & & \cdot \\ \cdot & & & \cdot \\ \alpha_{n} I_{m} \cdots & 0 & 0\end{array}\right]$

$B=\alpha(A) \tilde{B}^{\prime}$

for some $\tilde{B}^{\prime}$, where $\alpha_{i}$ are defined from

$$
\alpha(s)=\sum_{i=0}^{n} \alpha_{i} s^{i}=|s I-F|=0
$$

where $\alpha_{0}=1$ and

$$
\alpha(A)=\sum_{i=0}^{n} \alpha_{i} A^{i}
$$

Observe that $y, B, H, F$, and $A$ are specified with $\lambda(F) \neq \lambda(A) \tilde{B}$ is uniquely determined from $\tilde{B}^{\prime}=\alpha(A)^{-1} B$ and $T^{\prime}$ is also uniquely determined. If there are any common eigenvalues in $A$ and $F$ then $\tilde{B}$ and $T^{\prime}$ are clearly nonunique.

It might be thought that these equations can be employed to simplify the decision problem. This is indeed the case, however an even better reduction in complexity of the decision problem is achieved by exploiting properties of certain state space coordinate basis as discussed in the next section.

\section{IMMEDIATE SIMPLIFICATION OF THE Decision Probi.em}

In this section we point out that a suitable selection of coordinate basis for the observer and signal model yield immediately a substantial reduction in the number of unknowns in the observer decision problem and a total elimination of the inequalities.

\section{A. Coordinate Basis for the Observer}

Let $A$ be chosen as a direct sum of blocks

$$
\left[\begin{array}{cc}
0 & 1 \\
-v_{i}^{2}-\epsilon & -w_{i}^{2}-\epsilon
\end{array}\right]
$$

for the observer state dimension an even integer and the direct sum of such blocks with one "block" simply $-w_{i}^{2}$ - $\epsilon$ for the observer state dimension odd. Here $\epsilon$ is chosen as some small positive value to ensure that $\lambda_{j}[A]$ $<0$ for all $i$. With $A$ so chosen, the stability condition $C 1$ is automatically satisfied for all selection of $v_{i}$ and $w_{i}$. In other words, we have in one small stroke eliminated the inequalities from the conditions to be tested. The number of unknowns in $A$ is also reduced from $p^{2}$ to $p$. For convenience we use $\bar{A}$ to denote a vector consisting of $v_{i}$ and $w_{i}$ for all $i$.

Note that this choice of $A$ restricts the multiplicity of each eigenvalue to two. Further parameters must be included to search for an observer with higher order multiplicity of eigenvalues.

One further point to note is that should we desire to include the restriction that the observer has all real eigenvalues (so that it can be realized by a resistive capacitative network), $A$ could be chosen as the direct sum of "blocks" $-w_{i}^{2}-\epsilon$.

\section{B. Coordinate Basis for the Signal Model}

Since the signal model is assumed to be completely observable, without loss of generality, it may have the following form [8].

$$
\dot{x}(t)=\left[\begin{array}{ccc}
F_{11} F_{12} & \cdots & F_{1 m} \\
F_{21} F_{22} & & F_{1 m} \\
F_{m 1} F_{m 2} & \cdots & F_{m m}
\end{array}\right] x(t)+\left[\begin{array}{c}
G_{1} \\
G_{2} \\
\cdot \\
\cdot \\
G_{m}
\end{array}\right] u(t)
$$




$$
y(t)=H_{m m}^{-1}\left[H_{1}^{\prime} H_{2}^{\prime} \cdots H_{m}^{\prime}\right] x(t)
$$

with $H_{m m}$ some nonsingular matrix and

$$
\begin{gathered}
F_{i i}=\left[\begin{array}{ccccc}
0 & 0 & \cdots & 0 & x \\
1 & 0 & & 0 & x \\
0 & 1 & & 0 & x \\
\cdot & \cdot & & \cdot & \cdot \\
\cdot & \cdot & & \cdot & \cdot \\
\cdot & \cdot & & \cdot & \cdot \\
0 & 0 & & 1 & x
\end{array}\right], F_{i j}=\left[\begin{array}{cccc}
0 & 0 & 0 & x \\
0 & 0 & 0 & x \\
\cdot & & & \cdot \\
\cdot & & & \cdot \\
\cdot & & & \cdot \\
0 & 0 & 0 & x
\end{array}\right], \\
H_{i}=\left[\begin{array}{c}
0 \\
0 \\
\cdot \\
\cdot \\
e_{i}^{\prime}
\end{array}\right] .
\end{gathered}
$$

Here $F_{i j}$ is of dimension $\nu_{i} \times \nu_{j}$ for all $i$ and $j$ and structural indices $\left\{\nu_{1} \nu_{2} \cdots \nu_{m}\right\}$ where

$$
\sum_{i=1}^{n} \nu_{i}=n
$$

Also $x$ denotes an element which may be nonzero. The matrix $H_{i}$ is of dimension $\nu_{i} \times m$ and $e_{i}$ is an $m$-vector with unity $i$ th entry and otherwise having zero elements.

With the signal model (3.1)-(3.3), it is straightforward to derive simplified expressions for the conditions (2.3) and (2.4) of Lemma 2.1 starting out along the lines in [3]. First with the partitioning of $T^{\prime}$ into column vectors as

$$
T^{\prime}=\left[t_{1} t_{2} \cdots t_{n}\right]
$$

and the partitioning of $T^{\prime}$ and $K^{\prime}$ into $p \times \nu_{i}$ and $r \times \nu_{1}$ matrices as

$$
T^{\prime}=\left[T_{1}^{\prime} T_{2}^{\prime} \cdots T_{m}^{\prime}\right], K^{\prime}=\left[K_{1}^{\prime} K_{2}^{\prime} \cdots K_{m}^{\prime}\right] .
$$

Substitution of (3.4) into (2.4) yields the equalities for $i=1,2, \cdots m$

$$
T_{i}^{\prime}=\left[\tilde{t_{i}} A \tilde{t_{i}} A^{2} \tilde{t_{i}} \cdots A^{v_{i}-1} \tilde{t_{i}}\right]
$$

where $\tilde{t}_{1}=t_{1}, \tilde{t_{2}}=t_{\nu_{1}+1} ; \tilde{t_{3}}=t_{\nu_{1}+\nu_{2}+1}$, etc. Now a substitution of (3.5) into (2.3) yields the equalities for $i=1$, $2, \cdots m$

$$
K_{i}^{\prime}=C^{\prime} T^{\prime} \text { for } E=0, K_{i}^{\prime}=C^{\prime} T_{i}+E H_{i}^{\prime} \text { for } E \neq 0 .
$$

It now.proves convenient to define

$$
\tilde{T}^{\prime}=\left[\tilde{t}_{1} \tilde{t}_{2} \cdots \tilde{t}_{m}\right], K_{i}^{\prime}=\left[k(1, i) k(2, i) \cdots k\left(\nu_{i}, i\right)\right] .
$$

With these definitions for $k(j, i)$, we have that (3.7) [a version of (2.3)] implies the following condition.

Condition C2: The $r$-vectors $k(j, i)$ for $i=1,2, \cdots m$ and $j=1,2, \cdots \bar{\nu}_{i}$ satisfy

$$
k(j, i)=C^{\prime} A^{j-1} \tilde{t}_{i}
$$

where $\bar{\nu}_{i}=\nu_{i}$ for the case $E=0$, and $\nu_{i}=\bar{\nu}_{i}-1$ for the case $E \neq 0$.

Observe now that (3.4)-(3.6) are equivalent to the condition (2.4) if $B$ is defined by postmultiplication of $(2.4)$ by $H\left(H^{\prime} H\right)^{-1}$ to be $B=\left(T^{\prime} F-A T^{\prime}\right) H\left(H^{\prime} H\right)^{-1}$. Actually, using the canonical form for $H^{\prime},\left(H^{\prime} H\right)=I$ and the expression for $B$ is simply

$$
B=\left(T^{\prime} F-A T^{\prime}\right) H .
$$

In a similar manner postmultiplication of the condition (2.3b) by $H\left(H^{\prime} H\right)^{-1}$ yields an expression for $E$ as $E$ $=\left(K^{\prime}-C^{\prime} T^{\prime}\right) H\left(H^{\prime} H\right)^{-1}$ which for the canonical form case simplifies as

$$
E=\left(K^{\prime}-C^{\prime} T^{\prime}\right) H
$$

These expressions for $E$ together with (3.9) are equivalent statements of (3.7).

In summary then, working with the coordinate basis for signal model and observer as described in this subsection, instead of the quest for unknowns $A, C, B$, and $E$ to satisfy the conditions (2.3), (2.4), and C1 of Lemma 2.1, we have the less arduous quest for unknowns $\bar{A}, C$, and $\tilde{T}$ to satisfy Condition $\mathrm{C} 2$. Once suitable $\bar{A}, C$, and $\tilde{T}$ are determined, the matrices $B$ and $E$ are calculated explicitly from (3.10) and (3.11). In the next section, further simplifications to our Condition C2 are explored.

\section{Observer Properties}

In this section, some useful necessary conditions for the existence of minimal order, asymptotically stable observers are derived from the properties of such observers.

\section{A. Necessary Conditions on A}

The characteristic equation for any $A$ with negative eigenvalues may be written in the form

$\beta(s)=\prod_{i=1}^{n / 2}\left[s^{2}+s\left(w_{i}^{2}+\epsilon\right)+\left(v_{i}^{2}+\epsilon\right)\right]$ for $n$ even

$=\left(s+w_{n}^{2}+\epsilon\right) \prod_{i=1}^{(n-1) / 2}\left[s^{2}+s\left(w_{i}^{2}+\epsilon\right)+\left(v_{i}^{2}+\epsilon\right)\right]$ for $n$ odd

for $\epsilon$ some small positive constant.

With the aid of these equations it is a simple matter to express $\beta_{i}$, the coefficients of $\beta(s)$, as an explicit function of the vector $\bar{A}$ defined in Section III-A. We will use the notation $\beta_{i}(\bar{A})$ to denote this function. The CaleyHamilton theorem for the polynomial

$$
\beta(s)=\sum_{i=0}^{p} \beta_{i}(\bar{A})=|s I-A|=0
$$

is given by the equation

$$
\beta(A)=\sum_{i=0}^{p} \beta_{i}(\bar{A}) A^{i}=0
$$

Application of this theorem to (3.9) yields the following necessary condition on $\bar{A}$ (or on $\beta_{i}(\bar{A})$ ).

Condition $C 3$ : The vector $\bar{A}$ satisfies

$$
\sum_{l=0}^{p} \beta_{l}(\bar{A}) k(j+l, i)=0
$$


for $i=1,2, \cdots m$, and $j=1,2, \cdots\left(\bar{\nu}_{i}=q_{i}\right)$ where $q_{i}=\min$ $\left\{p, \bar{\nu}_{i}\right\}$. Note that since $k(\cdot, \cdot)$ is an $r$ vector there are $r$ $\sum_{i=1}^{m}\left(\bar{\nu}_{i}-q_{i}\right)$ conditions. That is, $r\left(n-\sum_{i=1}^{m} q_{i}\right)$ conditions for $E=0$ and $r\left(n-m-\sum_{i=1}^{m} q_{i}\right)$ conditions for $E \neq 0$.

\section{B. Restatement of Condition C2}

The equations of $\mathrm{C} 2$ may be written

$$
\left[k(1, i) k(2, i) \cdots k\left(\bar{\nu}_{i} i\right)\right]=C^{\prime}\left[\tilde{t}_{i} A \tilde{t}_{i} \cdots A^{\bar{\nu}} \dot{v}^{1} \tilde{t}_{i}\right]
$$

or $\bar{K}_{i}^{\prime}=C^{\prime} \bar{T}_{i}^{\prime}$ for all $i$.

Writing the characteristic equation of $A$ as $\beta(s)=s^{p}+$ $\beta_{p-1} s^{p-1} \cdots \beta_{1} s+\beta_{0}$ we may define the $\bar{\nu}_{i} \times\left(\bar{\nu}_{i}-p\right)$ matrix $S_{\beta}^{i}$ as

$\left(S_{\beta}^{i}\right) \triangleq\left[\begin{array}{cccccccccc}\beta_{0} & \beta_{1} & \beta_{2} & \cdots & \beta_{p-1} & 1 & 0 & \cdots & 0 & 0 \\ 0 & \beta_{0} & \beta_{1} & \cdots & \beta_{p-2} & \beta_{p-1} & 1 & \cdots & 0 & 0 \\ \cdot & \cdot & \cdot & & & & & & \cdot & \cdot \\ \cdot & \cdot & \cdot & & & & & & \cdot & \cdot \\ \cdot & \cdot & \cdot & & & & & & \cdot & \cdot \\ 0 & 0 & 0 & \cdots & \beta_{0} & \beta_{1} & \cdots & & \beta_{p-1} & 1\end{array}\right]$.

For the case $p \geqslant \bar{\nu}_{i}$ the matrix $\left[\begin{array}{c:c}S_{\beta}^{i} & I_{p} \\ & 0\end{array}\right]$ is nonsingular and (4.1) is equivalent to the following sets of equivalent conditions.

$$
{\overline{K_{i}}}^{\prime}\left[\begin{array}{c:c}
S_{\beta}^{i} & I_{p} \\
& 0
\end{array}\right]=C^{\prime} \bar{T}_{i}^{\prime}\left[\begin{array}{c:c}
S_{\beta}^{i} & I_{p} \\
& 0
\end{array}\right]
$$

or

$$
\bar{K}_{i}^{\prime} S_{\beta}^{i}=C^{\prime} \bar{T}_{i}^{\prime} S_{\beta}^{i} \quad \text { and } \quad \bar{K}_{i}^{\prime}\left[\begin{array}{c}
I_{p} \\
\overline{0}
\end{array}\right]=C^{\prime} \bar{T}_{i}^{\prime}\left[\begin{array}{c}
I_{p} \\
-0
\end{array}\right]
$$

or

$$
\bar{K}_{i}^{\prime} S_{\beta}^{i}=0 \quad \text { and } \quad k(j, i)=C^{\prime} A^{j-1} \tilde{t}_{1} \quad \text { for } \quad j=1,2, \cdots p
$$

Note that we have employed the Caley-Hamilton theorem $\sum_{i=0}^{p} \beta_{i} A^{i-1}=0$ which implies that $\bar{T}_{i}^{\prime} S_{\beta}^{i}=0$ for all $i$. For the case when $\beta(s)$ is chosen as in (4.1), the first of these conditions is equivalent to Condition C3. Now for the case $\bar{\nu}_{i}>p$ Condition C3 has no equations in $k(\cdot, i)$ and so (4.1) can not be simplified. Thus it is seen that Condition $\mathrm{C} 2$ is equivalent to $\mathrm{C} 3$ together with the condition

$$
k_{l}(j, i)=c_{l}^{\prime} A^{i-1} \tilde{t_{j}}
$$

for $l=1,2, \cdots r, i=1,2, \cdots q_{j}$, and $j=1,2, \cdots m$ where

$$
k(i, j)=\left[\begin{array}{c}
k_{1}(i, j) \\
k_{2}(i, j) \\
\cdot \\
\cdot \\
k_{r}(i, j)
\end{array}\right] C^{\prime}=\left[\begin{array}{c}
c_{1}^{\prime} \\
c_{2}^{\prime} \\
\cdot \\
\cdot \\
c_{r}^{\prime}
\end{array}\right]
$$

It proves convenient to introduce the following notation:

$$
\begin{aligned}
& \hat{T}^{\prime}=\left[\hat{T}_{1}^{\prime} \hat{T}_{2}^{\prime} \cdots \hat{T}_{m}^{\prime}\right], \hat{T}_{i}^{\prime}=\left[\tilde{t}_{i} A \tilde{t_{i}} \cdots A^{q_{i}-1_{t_{i}}}\right] \\
& \hat{K}^{\prime}=\left[\hat{K}_{1}^{\prime} \hat{K}_{2}^{\prime} \cdots \hat{K}_{m}^{\prime}\right], \hat{K}_{i}^{\prime}=\left[k(1, i) k(2, i) \cdots k\left(q_{i}, i\right)\right] \\
& D=\left[\begin{array}{c}
d_{1} \\
d_{2} \\
\cdot \\
\cdot \\
d_{m}
\end{array}\right], d_{j}=\left[\begin{array}{c}
k\left(q_{j}+1, j\right) \\
k\left(q_{j}+2, j\right) \\
\cdot \\
\cdot \\
k(p, j)
\end{array}\right] \\
& \tilde{K}^{\prime}=\left[\begin{array}{c}
\tilde{k_{1}} \\
\tilde{k}_{2} \\
\cdot \\
\tilde{k_{p}}
\end{array}\right], \tilde{k_{i}}=[k(i, 1) k(i, 2) \cdots k(i, m)] \text {. }
\end{aligned}
$$

Of course $\tilde{K}^{\prime}$ may well be a function of the unspecified matrix $D$ and should thus be written $\tilde{K}^{\prime}(D)$.

With the above notation and the assumption that $\mathrm{C} 3$ is satisfied, Condition $\mathrm{C} 2$ is equivalent to the following equivalent conditions.

Condition C4:

$$
\hat{K}^{\prime}=C^{\prime} \hat{T}^{\prime}
$$

Condition C5: There exists a matrix $D$ such that either of the following equivalent conditions is satisfied.

a) $\tilde{K}^{\prime}(D)=Q \tilde{T}^{\prime}$.

b) $\tilde{k_{i}}(D)=C A^{i-1} \tilde{T}^{\prime}$ for $i=1,2, \cdots p$.

c) The triple $\left\{C, A, \tilde{T}^{\prime}\right\}$ is a $p$ th-order realization with first $p$ Markov parameters $\tilde{k}_{1}(D), \tilde{k}_{2}(D) \cdots \tilde{k}_{p}(D)$.

\section{Necessary Condition on the Pair $\left[A, \tilde{T}^{\prime}\right]$}

We have seen in the above subsection that a necessary condition for an observer of order $p$ to exist is that there exist a $p$ th-order realization $\left\{C, A, \tilde{T}^{\prime}\right\}$ of the Markov parameters $k_{j}(D)(j=1,2, \cdots p)$ for some $D$. Clearly, a necessary condition for a minimal order observer to exist is that the realization $\left\{C, A, \tilde{T}^{\prime}\right\}$ be minimal or equivalently that

Condition $C 6$ : The pair $[A, C]$ is completely observable and the pair $\left[A, \tilde{T}^{\prime}\right]$ is completely controllable.

\section{Necessary Condition on $T$ and $\hat{T}$}

We now claim that the following conditions on $T$ and $\hat{T}$ are necessary conditions for a minimal order observer.

Condition C7: The matrix $\hat{T}^{\prime}$ is full rank.

To establish this claim we first consider certain controllability properties of the composite system consisting of the cascade of the signal model and observer as follows:

$$
\left[\begin{array}{c}
\dot{x} \\
\dot{y}
\end{array}\right]=\left[\begin{array}{cc}
F & 0 \\
B H^{\prime} & A
\end{array}\right]\left[\begin{array}{l}
x \\
y
\end{array}\right]+\left[\begin{array}{c}
G \\
T^{\prime} G
\end{array}\right]^{u} \triangleq \bar{F}\left[\begin{array}{l}
x \\
y
\end{array}\right]+\bar{G} u .
$$

The controllability matrix of this composite system is

$$
W=\left[\bar{G} \bar{F} \bar{G} \bar{F}^{2} \bar{G} \cdots \bar{F}^{n+p-1} \bar{G}\right]
$$




$$
W=\left[\begin{array}{l}
I_{n} \\
T^{\prime}
\end{array}\right]\left[G F G F^{2} G \cdots F^{n+p-1} G\right]
$$

where the latter equality is achieved by application of (2.4).

Now it is not difficult to see that in order for the observer to be minimal order the states $y$ of the above composite system (the observer states) must be completely controllable from the composite system input $u$. Equivalently the matrix $T^{\prime}\left[G F G \cdots F^{n+p-1} G\right]$ must have full rank $p$; and since $[F, G]$ is completely controllable this requires that $T^{\prime}$ have full rank. But rank $T=\operatorname{rank} \hat{T}$ since $\hat{T}^{\prime}$ is derived from $T^{\prime}$ merely be delection of some dependent columns. Thus a necessary condition for the observer to be minimal is that $T$ have full rank or equivalently that (4.7) be satisfied.

Notice that the Conditions C6 and C7 each imply that the pair $\left[A, T^{\prime}\right]$ is completely controllable.

\section{E. A Coordinate Basis for $A$}

Since $\left[A, \tilde{T}^{\prime}\right]$ is required to be completely controllable a suitable coordinate basis is described in [10] with structural indices $p_{1} p_{2} \cdots p_{m}$ not specified at this stage (see comment 1) next section).

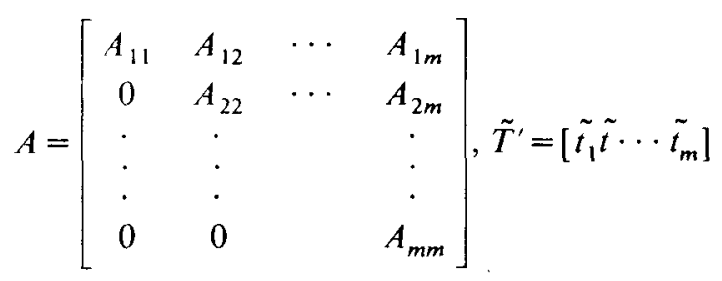

where

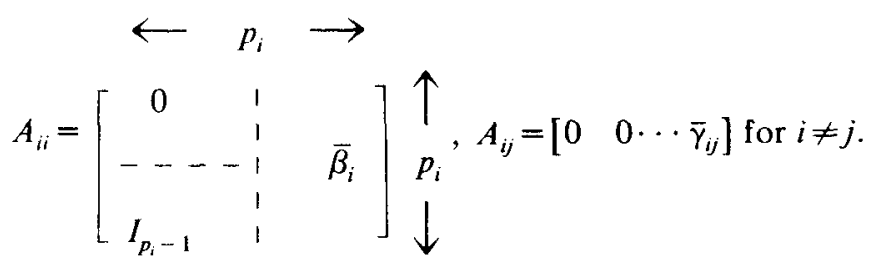

Also

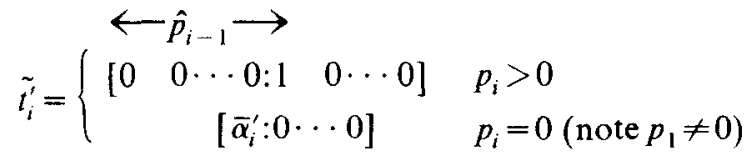

$$
\begin{aligned}
& \leftarrow \hat{p}_{i-1} \longrightarrow
\end{aligned}
$$

where

$$
\hat{p}_{i}=\sum_{j=1}^{i} p_{j}
$$

and $\alpha$ denotes a possible nonzero element. (The fact that the last $\left(p-\hat{p}_{i-1}\right)$ elements of $\tilde{t}_{i}^{\prime}$ are zero when $p_{i}=0$ is readily proven although not noted in [10]. Since $p_{i}=0$ we have that $\tilde{t_{i}}$ is dependent on the rows of the matrix $\left[\tilde{t}_{1} A \tilde{t}_{1} \cdots A^{p_{1}-1} \tilde{t}_{1}|\cdots| \tilde{t}_{i-1} A \tilde{t}_{i-1} \cdots A^{p_{i-1}-t_{t-1}}\right]=\left[I_{\hat{p}_{i-1}} 0\right]^{\prime}$ and our result is established.)
The unknown elements of $A, \tilde{T}$ comprise the vectors $\bar{\beta}_{i}$, $\bar{\gamma}_{i j}$ and $\bar{\alpha}_{i}$ for $i=1,2, \cdots m$ and $j=i+1, i+2, \cdots m$. Here $\bar{\beta}_{i}$ and $\bar{\gamma}_{i j}$ are $p_{i}$ vectors and $\bar{\alpha}_{i}$ is a $\hat{p}_{i-1}$ vector. There are in fact $\sum_{i=1}^{m}(m+1-i) p_{i}$ unknown elements in $A$ and $\tilde{T}$.

For the case when $A$ has left-half plane eigenvalues, $A_{i i}$ can be transformed to the form of Section III-A with $p_{i}$ unknowns denoted $\bar{A}_{i}$. That is $\bar{\beta}_{i}=\bar{\beta}_{i}\left(\bar{A}_{i}\right)$. Of course we have that

$$
\beta(s)=\prod_{i=1}^{m} \beta_{i i}(s)=0
$$

where $\beta_{i}(s)=0$ is the characteristic equation of $A_{i i}$ [with coefficients the elements of $\left.\bar{\beta}_{i}\left(\bar{A}_{i}\right)\right]$ and $\beta(s)=0$ is the characteristic equation of $A$ itself. Thus since $\bar{A}^{\prime}$ $=\left[\overline{A_{1}^{\prime}} \overline{A_{2}^{\prime}} \cdots \overline{A_{m}^{\prime}}\right]$, the coefficients $\beta_{l}(\bar{A})$ can be readily obtained from the coefficients $\beta_{i i}\left(\bar{A}_{i}\right)$ for all $i$.

With $A, \tilde{T}$ chosen in the form above, the matrix $\hat{T}$ may now be written

$$
\hat{T}^{\prime}=\left[\begin{array}{llll}
T_{11} & T_{12} & \cdots & T_{1 m} \\
0 & T_{22} & \cdots & T_{2 m} \\
& & & \cdot \\
0 & & \cdots & T_{m m}
\end{array}\right], \quad T_{i j} \text { is }\left(p_{i} \times q_{j}\right) \text { for all } i, j
$$

where for $p_{i} \geqslant q_{i}$

$$
T_{i i}=\left[\begin{array}{c}
I_{\bar{p}_{i}} \\
0
\end{array}\right] \text { and } T_{i j}=0_{p_{i} q_{j}}
$$

and for $p_{i} \leqslant q_{i}$

$$
T_{i i}=\left[I_{p_{i}}, X\right] T_{i j}=\left[0_{p_{i p j}} X\right] .
$$

Here $X$ denotes some possibly nonzero matrix of appropriate dimension.

\section{F. Further Minimal-Order Observer Properties}

First let us introduce the definitions.

Definitions: $\hat{T}_{S}^{\prime}, \hat{T}_{R}^{\prime}$. The submatrix $\hat{T}_{S}^{\prime}$ of $\hat{T}^{\prime}$ is obtained by deleting all the rows and columns of $\hat{T}^{\prime}$ containing any element of the blocks $I_{p_{i}}$ or $I_{\bar{p}_{i}}$ for all $i$. The submatrix $T_{R}^{\prime}$ of $T^{\prime}$ is obtained by deleting the columns and retaining only the rows of $\hat{T}^{\prime}$ with elements of the blocks $I_{p_{i}}$ or $I_{\bar{p}_{i}}$ for all $i$.

Now from Condition $\mathrm{C} 7$ and the above definition for $\hat{T}_{s}^{\prime}$ we have the following.

Condition $C 8: \hat{T}_{S}$ has full rank $\left(p-\sum_{i=1}^{m} \eta_{i}\right)$ or equivalently $\left|T_{S}^{\prime} T_{S}\right|>0$.

Also, a necessary condition for $\mathrm{C} 8$ to be satisfied is that

$$
\sum_{j=0}^{i}\left(q_{m-j}-\eta_{m-j}\right) \geqslant \sum_{j=0}^{i}\left(p_{m-j}-\eta_{m-j}\right)
$$

for all $i$ or more simply the following. 
Condition C9:

$$
\sum_{j=0}^{i} q_{m-j} \geqslant \sum_{j=0}^{i} p_{m-j}
$$

for $i=0,1, \cdots M-1$.

In order to derive an alternative condition to $\mathrm{C} 4$ in terms of $\hat{T}_{S}$, we introduce the further notations

$$
\begin{aligned}
& \leftarrow q_{1} \rightarrow \leftarrow q_{2} \rightarrow \\
& \leftarrow \eta_{1} \rightarrow \leftarrow \eta_{2} \rightarrow \\
\hat{K}^{\prime}= & {\left[\hat{K}_{1 a}^{\prime}\left|\hat{K}_{1 b}^{\prime}\right| \hat{K}_{2 a}^{\prime}\left|\hat{K}_{2 b}^{\prime}\right| \cdots\left|\hat{K}_{m a}^{\prime}\right| \hat{K}_{m b}^{\prime}\right] } \\
C^{\prime}= & {\left[C_{1 a}^{\prime}\left|C_{1 b}^{\prime}\right| \quad C_{2 a}^{\prime}\left|C_{2 b}^{\prime}\right| \cdots\left|C_{m a}^{\prime}\right| C_{m b}^{\prime}\right] } \\
& \leftarrow \eta_{1} \rightarrow \leftarrow \eta_{2} \rightarrow \\
& \leftarrow p_{1} \rightarrow \leftarrow p_{2} \rightarrow \\
\hat{K}_{a}^{\prime}= & {\left[\hat{K}_{1 a}^{\prime} \hat{K}_{2 a}^{\prime} \cdots \hat{K}_{m a}^{\prime}\right], \quad \hat{K}_{b}^{\prime}=\left[\hat{K}_{1 b}^{\prime} \hat{K}_{2 b}^{\prime} \cdots \hat{K}_{m b}^{\prime}\right] } \\
\hat{C}_{a}^{\prime}= & {\left[C_{1 a}^{\prime} C_{2 a}^{\prime} \cdots C_{m a}^{\prime}\right], \quad C_{b}^{\prime}=\left[C_{1 b}^{\prime} C_{2 b}^{\prime} \cdots C_{m b}^{\prime}\right] }
\end{aligned}
$$

where $\eta_{i}=\min \left(p_{i}, q_{i}\right)=\min \left(p_{i}, \bar{v}_{i}\right)$

With the above notation Condition $\mathrm{C} 4$ can be expressed as follows.

Condition ClO:

$$
C_{a}^{\prime}=\hat{K}_{a}^{\prime}, \hat{K}_{b}^{\prime}=\hat{K}_{a}^{\prime} \hat{T}_{R}^{\prime}+C_{b}^{\prime} T_{s}^{\prime}
$$

\section{G. A Lower Bound on the Order of the Minimal Observer}

A study of the minimum possible rank of the product of the observability matrix $Q$ [of (2.7)] and what may be termed a controllability matrix $T^{\prime}$, allows us to readily calculate a lower bound on the order of an observer. Decision methods are not required in the calculation.

Let the minimum rank of $Q T^{\prime}$ be $p_{\min }$, then it is clear that both $Q$ and $T^{\prime}$ must have rank greater than or equal to $p_{\min }$ and thus any observer satisfying the appropriate controllability and observability conditions mentioned in the earlier lemmas, must be of order no less than $p_{\min }$. (Recall that the rank of $Q$ and the rank of $T^{\prime}$ must each be $p$ where $p$ is the order of a minimal-order observer.) Thus it remains to give a procedure for calculation of $p_{\min }$.

An expansion for $Q T^{\prime}$ is given as

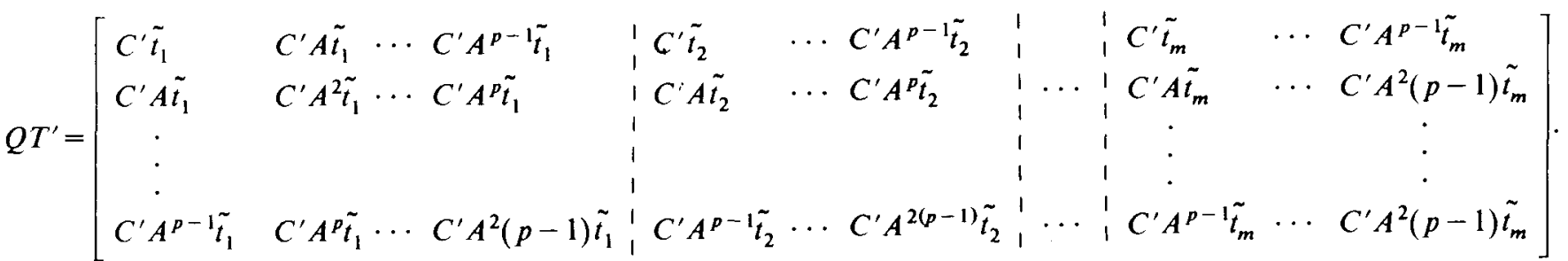

The first point to observe is that many of the elements of this matrix are specified quantities $k(j, i)$ for $j=1,2, \cdots q_{i}$ and $i=1,2, \cdots m$, while the remainder are dependent on unknown parameters earlier denoted $D$. The expression for $Q T^{\prime}$ is now rewritten in terms of the known $r$ vectors $k(j, i)$ and " $X$ " indicating unspecified elements as follows:

$$
Q T^{\prime}=\left[R_{1} R_{2} \cdots R_{M}\right]
$$

where $R_{i}$ is the $p$ th leading principal submatrix of

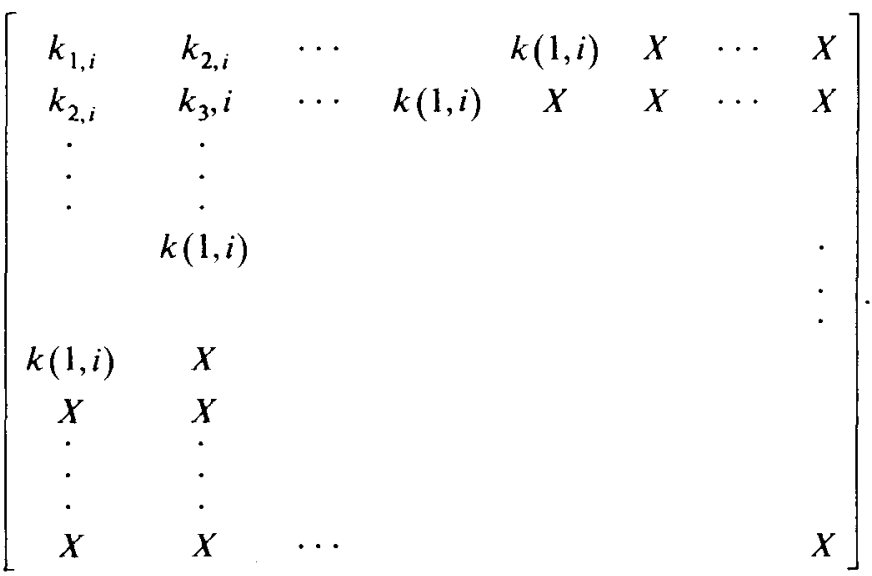

The minimum possible rank of such a matrix turns out to be the number of rows of the matrix $Q T^{\prime}$ which (for all possible unknown elements $X$ ) are independent. To test whether or not the $i$ th row is independent of rows $j=1$, $2, \cdots i-1$ for all possible unknown elements, it suffices to delete all columns of $Q T^{\prime}$ containing the unknown elements of the $i$ th row and then apply standard techniques.

We conclude that testing the rank of $Q T^{\prime}$ as given in (4.12) yields a lower bound $p_{\min }$ for the order of a minimal observer of $K^{\prime} x$. Thus instead of testing for observer orders $0,1,2, \cdots$ it is sufficient to test for observers of order $p_{\text {min }}, p_{\text {min }}+1, p_{\text {min }}+2, \cdots$, until the necessary and sufficient conditions of the lemmas are satisfied.

\section{Computing a Minimal-Order Observer}

In this section, the decision problem which arises in the minimal-order observer design procedures is further simplified. It appears that the simplifications are as much as can be achieved. Actually, two sets of useful existence conditions can be established, depending on whether we work with $\left[A, \tilde{T}^{\prime}\right]$ in the completely controllable form

(4.14) or with $[A, C]$ in the dual observable form.

\section{A. $[A, \tilde{T}]$ in Controllable Form}

Here two cases derived from $\mathrm{C} 8$ and $\mathrm{C} 10$ are worth giving separate treatment. 
Condition C11: 1) For the case when $p_{i} \leqslant \bar{\nu}_{i}$ (and thus $\eta_{i}-p_{i}$ ) for all $i$, then

$$
C_{a}^{\prime}=\hat{K}_{a}^{\prime}, \hat{K}_{b}^{\prime}=\hat{K}_{a}^{\prime} \hat{T}_{R}^{\prime}
$$

2) For the case when $p_{i}>\bar{\nu}_{i}$ (and thus $\left(\eta_{i}<p_{i}\right)$ ) for some $i$, then

$$
\begin{aligned}
& C_{a}^{\prime}=\hat{K}_{a}^{\prime}, C_{b}^{\prime}=\left[\hat{K}_{b}^{\prime}-\hat{K}_{a}^{\prime} T_{R}^{\prime}\right] T_{S}\left(T_{S}^{\prime} T_{S}^{\prime}\right)^{-1} \\
& {\left[\hat{K}_{b}^{\prime}-\hat{K}_{a}^{\prime} T_{R}^{\prime}\right]\left[T_{S}\left(T_{S}^{\prime} T_{S}\right)^{A} T_{S}^{\prime}-\left|T_{S}^{\prime} T_{S}^{\prime}\right|\right]=0} \\
& \left|T_{S}^{\prime} T_{S}\right|>0
\end{aligned}
$$

where $(X)^{A}$ denotes the adjoint of $X$.

We are now in a position to state one of the key theorems of this paper.

Theorems 5.1: For the signal model (3.4), (3.5) (where $H_{m m}=I_{m}$ ), a necessary and sufficient condition for the existence of a completely controllable asymptotically stable $p$ th-order observer of $K^{\prime} x$ is that there exist for some set of structural indices $p_{i}$ satisfying $C 9$, and $\sum_{i=1}^{m}(m+$ $1-i) p_{i}$ unknown parameters $\left\{\bar{A}_{i}, \bar{\gamma}_{i}, \bar{\alpha}_{i}\right\}$ for $i=1,2, \cdots m$ (defined in Section III-E) such that Conditions C3 and C11 are satisfied.

Comments: 1) Since $\mathrm{C} 3$ and $\mathrm{C} 11$ are multivariable polynomial equalities (and one inequality which is readily converted to an equality by the addition of one state variable), the decision methods of [5] and [7] may be applied for $p=p_{\min }, p_{\min }+1, \cdots$ for all possible $p_{i}$ satisfying $C 9$, until it is determined that a solution of $\mathrm{C} 3$ and C11 exists. The methods of [5] and [7] can then be applied to yield suitable parameters $\left\{\bar{A}_{i}, \bar{\gamma}_{i}, \bar{\alpha}_{i}\right\}$ from which $A, T$, $C, B$ (and $E$ ) can be determined, [via (4.14), (5.2), (3.8) (and (3.10))].

2) In the event that $p_{i} \leqslant \nu_{i}$ for all $i$, it is clear that Condition $\mathrm{Cll}$ is considerably simpler to test than when $p_{i}>v_{i}$ for some $v_{i}$.

3) The decision problem is stated above is considerably simpler than that stated in Section III. There is a reduction from $[p+p(m+r)]$ unknowns to $\sum_{i=1}^{m}(m+1-i) p_{i}$ $\leqslant p m$ unknowns here. Also the number of equalities is reduced, although the number of tests required is increased. Since there are a number of restrictions on $p_{i}$, the number $\left(\begin{array}{c}p+m-1 \\ p\end{array}\right)$ will represent on upper bound on the number of tests required, where $\left(\begin{array}{l}x \\ y\end{array}\right)=\frac{x !}{y !(x-y) !}$.

4) Notice that for the scalar output case $m=1, p=p_{1}$, $\nu_{1}=n$, and for $p<n-1$, we have $\eta_{1}=q_{1}=p$ and thus $\hat{T}_{S}^{\prime}$, $\hat{T}_{k}^{\prime}, \hat{K}_{b}$, and $C_{b}^{\prime}$ are of zero order. Thus Condition C11 is always satisfied. For this case then, there is a considerable simplification of the above lemma.

5) So far in our developments, we have assumed a fixed set of structural indices $\nu_{i}$ for the signal model. Clearly, Conditions C3 and C11 may be more complicated with one set of structural indices than with another set, but this requires a tedious separate study.

\section{B. $[A, C]$ in Observable Form}

In this subsection, we derive an alternative result to Lemma 5.1 by working with $[A, C]$ in the observable form corresponding to the controllable form (4.14) for $\left[A, \tilde{T}^{\prime}\right]$. In this instance additional unknowns which are a subset of $D$ are involved and unhappily the algebra is quite different than for the previous case studied.

Let us assume for the remainder of the section that the pair $[A, C]$ is in the observable form corresponding to the controllable form (4.14) for $\left[A, T^{\prime}\right]$. Then for specified structural indices $p_{i}$ where

$$
\sum_{l=1}^{r} p_{i}=p
$$

then there are $\sum_{l=1}^{r}(r+1-i) p_{i}$ unknown elements in $[A, C]$. The matrix $\tilde{Q}$ and its left inverse $\tilde{Q}^{L}$ (in that $\tilde{Q}^{L} \tilde{Q}=I$ ) have the following forms:

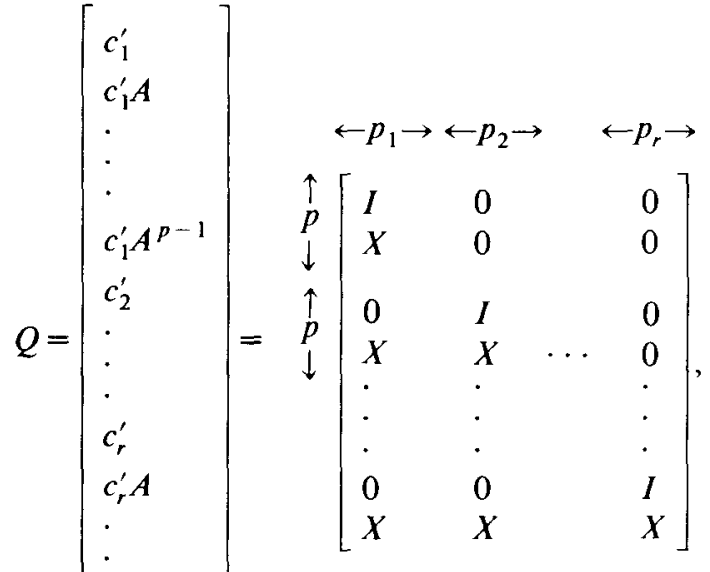

$$
\begin{aligned}
& \left.c_{r}^{\prime} A^{p-1}\right] \\
& Q^{L}=\left[\begin{array}{cccccc}
I & 0 & 0 & 0 & 0 & 0 \\
& & & & & \\
0 & 0 & I & 0 & 0 & 0 \\
& & & & & \\
& & & & & \\
0 & 0 & 0 & 0 & I & 0
\end{array}\right] \begin{array}{c}
\uparrow \\
p_{1} \\
\downarrow \\
p_{2} \\
\downarrow \\
\uparrow \\
p_{r}
\end{array}
\end{aligned}
$$

With these definitions, a consequence of Condition $\mathrm{C} 5$ are the relationships for $j=1,2, \cdots m$

$$
\begin{aligned}
t_{j}^{\prime}=\left[k_{1}^{\prime}(i, j) k_{1}^{\prime}(2, j) \cdots k_{1}^{\prime}\left(p_{1}, j\right) \mid\right. & \cdots \\
& \left.\mid k_{r}^{\prime}(1, j) k_{r}^{\prime}(2, j) \cdots k_{r}^{\prime}\left(p_{r}, j\right)\right] .
\end{aligned}
$$

Recall that the elements $k_{l}(i, j)$ for $i=q_{j}+1, q_{j}+2, \cdots p_{l}$ and $l=1,2, \cdots r$ are unspecified. Thus with $\eta_{l_{j}}=\min$ 
$\left\{p_{l}, q_{j}\right\}$ there are a total of $\sum_{j=1}^{m} \sum_{l=1}^{r}\left(p_{l}-\eta_{l j}\right)$ unspecified elements in $\tilde{T}^{\prime}$.

It is now not difficult to show that with $\tilde{T}^{\prime}$ defined in terms of the elements $k_{l}(i, j)$ as above, condition (4.2) simplifies to the constraint

$$
k_{l}(i, j)=c_{l}^{\prime} A^{i-1} \tilde{t_{j}}
$$

for $j=1,2, \cdots m, l=1,2, \cdots r$ and $\eta=p_{l}+1, p_{l}+2 \cdots q_{j}$. With $i$ so constrained we have that [see the appropriate submatrix of $\tilde{Q}$ in (5.1)]

$$
\begin{gathered}
p_{1} p_{2} \quad p_{l} \\
c_{l}^{\prime} A^{i-1}=[X|X| \cdots|X| 0] .
\end{gathered}
$$

Substitution of (5.4) into (5.3) points up the fact that not all the unspecified elements of $\tilde{t}_{j}$ are involved in the constraint (5.3). For a specified $j$, there is some maximum $l$ [denoted $l_{j}(\max )$ ] such that $p_{l_{j}}(\max )<q_{j}$. Using this notation the unspecified elements of $\tilde{t}_{j}$ unsolved in the constraint $(5.3)$ and $k_{l}(i, j)$ for $j=1,2, \cdots m, l=1,2, \cdots$ $\left[l_{j}(\max )-1\right]$ and $i=q_{j}+1, q_{j}+2 \cdots p_{l}$. The number of such unspecified elements in $\sum_{j=1}^{m} \sum_{j=1}^{l_{j}(\max )-1}\left(p_{l}-\eta_{l_{j}}\right)$. Thus we have the following theorem.

Theorem 5.2: For the signal model (3.4), (3.5) (where $H_{m m}=I_{m}$ ), a necessary and sufficient condition for the existence of a completely observable $p$ th-order observer which is asymptotically stable, is that there exist structural indices $p_{i}$, and $\sum_{l=1}^{r}(r+1-i) p_{i}$ fundamental parameters of the pair $[A, C]$ in the Bucy observable form [10] (corresponding to the controllable form of Section IV) (for $j=1$, $\left.2, \cdots m, l=1,2, \cdots l_{j}(\max )-1\right)$ and $i=q_{j}+1, q_{j}+2, \cdots p_{l}$ such that Condition C3 and Condition (5.4) are satisfied.

Comments: 1) With parameters satisfying the conditions of the above theorem, $\tilde{T}$ is given (5.2) and the remaining observer parameters determined from definitions given earlier.

2) Once again, the above existence conditions can be tested using the decision methods of [7]. The number of unknown elements in the existence conditions is

$$
\sum_{l=1}^{r}(r+1+l) p_{l}+\sum_{j=1}^{m} \sum_{l=1}^{l_{j}(\max )-1}\left(p_{l}-\eta_{l_{j}}\right)
$$

which for $r<m$ may well be less than the number of unknowns in Lemma 5.1. For the case when the $p_{i}$ increase monotonically with $i$ the number of unknowns in $\tilde{T}^{\prime}$ reduces to zero.

3) Since the (5.4) is a linear function of the unknown $\tilde{T}^{\prime}$ it is possible, at least in theory, to reduce the number of such unknowns by the number of independent equations of (5.3) solving these parameters. In general, these independent equations cannot be identified except by trial and error, and thus any such parameter reduction would involve numerous repetition of the decision problem. The number of sets of possible structural indices is $\left(\begin{array}{c}p+r-1 \\ p\end{array}\right)$, which represents the number of decision problems to be examined to show that no $p$ th-order observer may exist.

4) For the scalar estimate case when $r=1$, the number of unknown elements in the constraint equations reduces to $p$, and the conditions (5.3) evanesees. This result is possibly the most useful new result of the paper.

\section{Conclusions}

Although we have simplified considerably the decision problem arising from the minimal observer problem when observing $K^{\prime} x$, there is still a "curse of dimensionality" or exponential effect on computational complexity which would make certain minimal observer problems for observing some $K^{\prime} x$ intractable. Perhaps the most useful result of the paper is the comment 3 ) of Theorem 5.2 for this points out that in the very common scalar estimate case $\left(K^{\prime} x\right.$ is scalar), the necessary and sufficient conditions involve only $p$ unknowns where $p \leqslant n-m$ and $p$ is the observer order. For a large number of outputs, $m$, available (a frequent situation) the value of $p$ may well be small even for high-order signal models and the decision problem quite tractable. For the case when there is a scalar measurement $(m=1)$, the situation is not quite so attractive since $p \leqslant n-1$, and with less outputs there is less likelihood of a low-order observer. Unfortunately, there does not appear to be necessary and sufficient conditions for other than the $m=1$ and $r=1$ cases, involving only $p$ unknown parameters, and so the cases where $m \neq 1$ and $r \neq 1$ are inevitably more complicated to work with.

One final point is that the various results in Section III of the present paper have application to the determination of minimal stable realizations from partially specified Markov parameters (the duality was first pointed out by T.E. Fortmann). This topic is explored in a companion paper [9].

\section{REFERENCES}

[1] B. D. O. Anderson and J. B. Moore, Linear Optimal Control. Englewood Cliffs, N. J.: Prentice-Hall, 1971.

[2] D. G. Luenberger, "Observers for multivariable systems," IEEE Trans. Automat. Contr., vol. AC-11, pp. 190-197, Apr. 1966.

[3] T. E. Fortmann and D. Williamson, "Design of low-order observers for linear feedback control laws," IEEE Trans. Automat. Contr., vol. AC-17, pp. 301-308, June 1972.

[4] J. B. Moore, "A note on minimal order observers," IEEE Trans. Automat. Contr. (Tech. Notes and Corresp.), vol. AC-17, pp. 255 256, Apr. 1972.

[5] B. D. O. Anderson, N. K. Bose, and E. I. Jury, "Output feedback stabilization and related problems-Solution via decision methods," IEEE Trans. Automat. Contr., vol. AC-20, pp. 53-65, Feb. 1975.

[6] A. Tarski, A Decision Method for Elementary Algebra and Geometry. Berkeley, Calif.: Univ. Calif. Press, 1951.

[7] A. Seidenberg, "A new decision method for elementary algebra," Ann. Math., vol. 60, pp. 365-374, 1954.

[8] D. G. Luenberger, "Canonical forms for linear multivariable systems," IEEE Trans. Automat. Contr. (Short Papers), vol. AC-12, pp. 290-293, June 1967. 
[9] G. Ledwich and J. B. Moore, "On minimal stable realizations with partially specified Markov parameters," in Proc. 1975 Int. Fed. Automat. Contr. Conf., Boston, Mass.

[10] J. E. Ackermann and R. S. Bucy, "Canonical minimal realization of a matrix of impulse response sequences," Inform. Contr., vol. 19, pp. 224-231, 1971 .

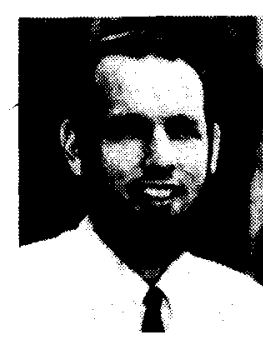

John B. Moore (S'66-M'67) was born in China in 1941. He received the B.S., M.S., and Ph.D. degrees in electrical engineering from the University of Santa Clara, Santa Clara, Calif., in 1963,1964 , and 1967 , respectively. He was appointed Senior Lecturer at the University of Newcastle, Newcastle, New South Wales, Australia, in 1968 and Full Professor in 1973.

He has held other visiting academic appointments at the University of Santa Clara, in 1968 the University of Maryland, College Park, in

1970, Colorado State University, Fort Collins, and Imperial College,
London, England, both in 1974. He has spent periods in industry as a Design Engineer and as a Consultant. $\mathrm{He}$ is co-author, with B.D.O. Anderson, of Linear Optimal Control (Englewood Cliffs, N. J.: PrenticeHall). Dr. Moore's current research in control and communication systems is supported by the Australian Research Grants Committee and the Radio Research Board.

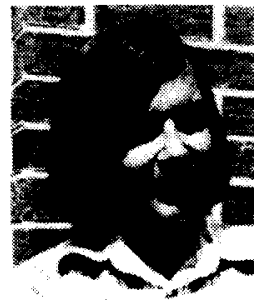

Gerard F. Ledwich (S'74) was born in Melbourne, Australia, on December 17, 1951. He received the B.E. degree in electrical engineering from the University of Queensland, Brisbane, Queensland, Australia, in 1973. He is currently completing the requirements for the Ph.D. degree in electrical engineering at the University of Newcastle, Newcastle, New South Wales, Australia. 